\title{
Retroperitoneal Actinomycosis: A Rare Sequela of an Infected Obstructing Ureteral Stone
}

\author{
Elizabeth Bearrick, BS, Colby A. Dixon, MD, Joshua Rhein, MD,2 and Michael S. Borofsky, MD¹
}

\begin{abstract}
Background: Actinomycosis is a condition in which Actinomyces, a normal component of the oral and gastrointenstial flora, becomes pathogenic in the setting of damaged tissue, leading to widespread tissue destruction across fascial planes. Prior literature describing this condition is rare, particularly cases involving the retroperitoneum. In this study, we report a case of retroperitoneal actinomycosis caused by an infected, obstructing ureteral stone.

Case Presentation: A 48-year-old woman with a history of substance abuse, malnutrition, and gastric bypass presented to the emergency room with a 3-week history of abdominal pain and fevers. Workup revealed a $9 \mathrm{~mm}$ obstructing right ureteral stone with associated perinephric fluid collection that was concerning for forniceal rupture. There was left hydronephrosis and a $3 \mathrm{~mm}$ lower pole renal calculus as well. The patient underwent emergent decompression where bilateral duplicated collecting systems were identified, requiring stenting of all four moieties to ensure maximal decompression in the setting of obstructive pyelonephritis. Urine cultures grew Escherichia coli and Candida. The patient continued to deteriorate despite culture appropriate antibiotic therapy; repeat scan revealed progression of her perinephric fluid collection into a loculated retroperitoneal abscess. A percutaneous drain was placed, and nearly half a liter of pus was evacuated. Fluid cultures grew Actinomyces, and she ultimately recovered after a prolonged course of antibiotics, including 1 month of intravenous therapy and an additional 6 months of oral treatment. All stones were ultimately removed via ureteroscopy.

Conclusion: Actinomycosis is a rare invasive infection that is caused when the Actinomyces bacteria colonizes damaged tissue. We present the first reported case of urolithiasis inciting this process via tissue damage caused by obstruction and infection. Although rare, heightened suspicion is warranted among immunocompromised hosts who do not improve after decompression in such scenarios.
\end{abstract}

Keywords: ureteral stones, infection/inflammation, urolithiasis, ureteroscopy, actinomycosis, pyelonephritis, retroperitoneum

\section{Clinical History}

A 48-YEAR-OLD WOMAN presented to a tertiary care medical center emergency room with a 3-week history of lower abdominal pain, dysuria, and subjective fevers and chills. Her past medical history was significant for prior Roux-en-Y gastric bypass with liver failure and polysubstance abuse, resulting in chronic malnutrition (prealbumin of $2.0 \mathrm{mg} / \mathrm{dL}$ ). Work-up in the emergency room was notable for leukocytosis to $19 \times 10^{9}$ cells/L, acute kidney injury with creatinine elevation to $1.93 \mathrm{mg} / \mathrm{dL}$ (baseline $0.7 \mathrm{mg} / \mathrm{dL}$ ), and urinalysis suggesting infection with large leukocyte esterase, moderate blood, and many bacteria.

\section{Physical Exam}

Vital signs were notable for a temperature of $98.4^{\circ} \mathrm{F}$, blood pressure of $95 / 57 \mathrm{~m}$, and heart rate of 79 beats per

\footnotetext{
${ }^{1}$ Department of Urology, University of Minnesota Medical School, Minneapolis, Minnesota.

${ }^{2}$ Division of Infectious Diseases and International Medicine, Department of Medicine, University of Minnesota Medical School, Minneapolis, Minnesota.

(C) Elizabeth Bearrick et al. 2017; Published by Mary Ann Liebert, Inc. This is an Open Access article distributed under the terms of the Creative Commons Attribution License, which permits unrestricted use, distribution, and reproduction in any medium, provided the original work is properly cited.
} 
minute. She was noted to have bilateral costovertebral angle tenderness worse on the right side. The abdomen was otherwise soft.

\section{Diagnosis}

She was admitted to the medical service for presumed pyelonephritis and was started on intravenous (IV) levofloxacin, but had no improvement in symptoms. By hospital day 2, she continued to have fevers with increased leukocytosis to $39 \times 10^{9}$ cells/L and antibiotics were broadened to meropenem. Preliminary urine cultures grew $>100,000 \mathrm{CFU} / \mathrm{mL}$ of pansensitive Escherichia coli and given the lack of other symptoms, she was transitioned back to levofloxacin. Renal ultrasound at this time was suggestive of pyelonephritis, with no renal abscess or hydronephrosis. Despite ongoing IV antibiotic therapy that was effective for presumed $E$. coli, she continued to have fevers and leukocytosis and, ultimately, underwent a CT abdomen/pelvis that showed a $9 \mathrm{~mm}$ obstructing stone in the mid right ureter, a $3 \mathrm{~mm}$ left lower pole stone, mild bilateral hydronephrosis, and a $9 \times 11 \mathrm{~cm}$ right perinephric fluid collection that was concerning for forniceal rupture.

\section{Intervention}

The patient was subsequently taken to the operating room for emergent ureteral stent placement. Cystoscopy with ret- rograde pyelogram was notable for complete bilateral duplicated collecting systems, and Double-J stents were placed in all four moieties for maximal decompression in the setting of obstructive pyelonephritis (Fig. 1). Urine cultures were sent from her renal pelvices, which ultimately grew Candida albicans/dubliniensis. Despite ureteral stent placement, she continued to have fevers, hypotension, and leukocytosis to $30 \times 10^{9}$ cells/L 48 hours after decompression. A repeat CT scan was obtained, which demonstrated progression of her previously visualized right retroperitoneal fluid collection into a loculated, rim-enhancing collection that was concerning for abscess (Fig. 2). A percutaneous drain was placed into the abscess by interventional radiology with drainage of $>300$ cc of purulent fluid. A gram stain of the fluid demonstrated many polymorphonuclear leukocytes and grampositive cocci that did not grow in culture. Day 2 cultures from this fluid ultimately revealed growth of Actinomyces.

\section{Follow-Up and Outcomes}

Based on the patient's culture results, she was treated with a 4-week course of continuous infusion of IV penicillin $G$ followed by a 6-month course of oral penicillin V. A repeat CT scan at 3 weeks revealed resolution of the collection, and the drain was removed. Ureteroscopy was performed 1 month later while the patient was on antibiotics and antifungals, and
FIG. 1. CT scan with demonstration of right-sided hydronephrosis and perinephric stranding (top left), right ureteral stone designated by arrow (top right), left lower pole renal stone designated by arrow (bottom left), and retrograde pyelogram with bilateral duplicated collecting systems (bottom right).

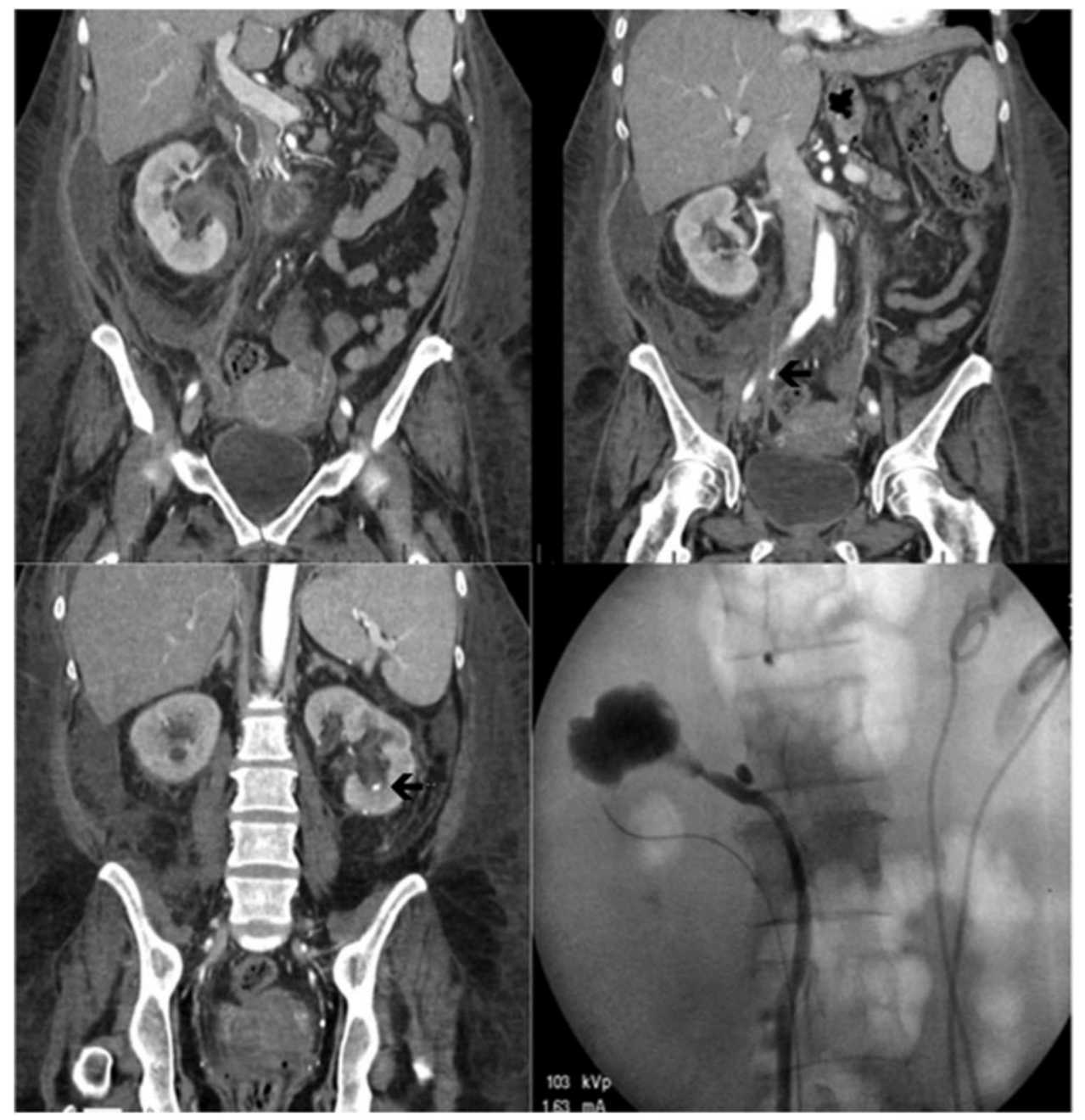




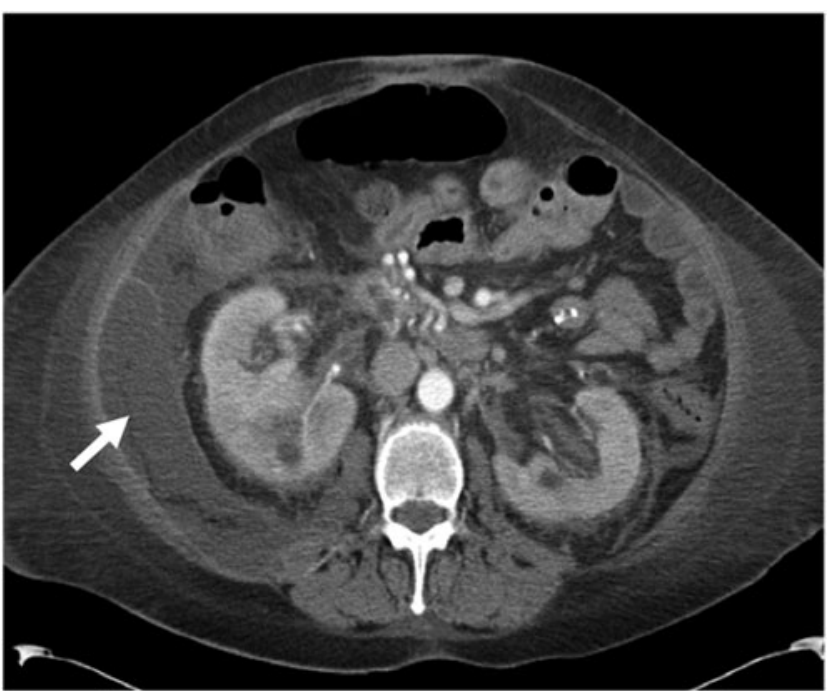

FIG. 2. Demonstration of large, loculated rim enhancing right retroperitoneal fluid collection (arrow).

the right ureteral as well as left renal stones were removed. They were noted to be exceptionally fragile and broke into pieces on manipulation with the basket. Stone analysis on several small pieces of the initial stone returned as organic matter, and stone culture grew yeast ( $C$. albicans/ dubliniensis, Candida kefyr) raising suspicion for either an inaccurate analysis or possibly a matrix stone. Antibiotic and antifungal therapy was continued, and stents were removed 1 week after surgery. The patient ultimately made a full recovery from her acute infectious illness.

\section{Discussion}

Actinomycosis is an infectious condition in which body tissues have been invaded by bacteria of the Actinomyces genus, most commonly Actinomyces israelii. Such bacteria are commonly part of normal oral, gastrointestinal, and genitourinary flora but can become pathogenic under select conditions. ${ }^{1}$ Actinomyces is a gram-positive, filamentous anaerobic bacteria. Though difficult to isolate in culture media, it is classically diagnosed on gram stain and confirmed by the presence of characteristic "sulfur granules": round, yellow particles $1 \mathrm{~mm}$ in diameter that contain colonies of Actinomyces mixed with polysaccharide complexes that are designed to prevent host phagocytosis. ${ }^{1}$

Unlike many other pathogens, Actinomyces typically does not penetrate normal mucosa and becomes pathogenic only in the presence of damaged or necrotic tissue. Further, as Actinomyces abscesses progress, they can lead to widespread disruption of tissue planes with subsequent abscess and fistula formation. Predisposing factors to actinomycosis include various forms of tissue injury, including history of penetrating trauma, intestinal necrosis, intraabdominal devices such as intrauterine devices (IUD), and immunosuppressive conditions such as diabetes mellitus, chronic steroid therapy, or chronic inflammatory disease.

Our patient had numerous conditions predisposing her to poor tissue health, most notably severe malnutrition in the setting of gastrointestinal bypass, liver failure, and polysubstance abuse. She did have a history of IUD for many years as well, and although this had been removed several years before her presentation, the presence of an IUD is a known colonizing factor for the Actinomyces organism.

We suspect that in this case, the presence of an obstructing ureteral stone with coexisting pyelonephritis (E. coli and Candida) in the setting of a relatively immunocompromised host caused sufficient tissue damage to allow for colonization with Actinomyces. Unlike more typical pathogens, including E. coli and Candida that also colonized her urine, Actinomyces is unique in its ability to invade fascial planes. This may explain why only Actinomyces and none of the other involved organisms were grown out of her abscess fluid. Of note, gram-positive cocci were also observed on gram stain of fluid. Actinomyces infections almost always contain other concomitant aerobic and/or anaerobic bacterial species, which may act as synergistic pathogens to strengthen the comparatively low invasive power of Actinomyces. ${ }^{1}$ In this case, we suspect that the gram-positive cocci observed on gram stain could have indicated a mixed infection with an anaerobic species that was resistant to levofloxacin. Other "companion" organisms may have been present from the onset of symptoms but were susceptible to levofloxacin and unable to be cultured after receiving therapy. Alternatively, urine leakage via forniceal extravasation could have provided an outlet for bacterial invasion into the retroperitoneum, though it would not entirely explain the evolution and timing of abscess formation or why Actinomyces and not E. coli or Candida were detected from the abscess drainage.

Actinomycosis is a rare disease. For perspective, the largest multicenter review of actinomycoses to date found only 28 total cases of actinomycoses across six tertiary medical centers in France over a 14-year period. ${ }^{2}$ Of these cases, only 9 were abdominopelvic in origin. Our case is particularly unique as case reports of actinomycosis developing primarily in the kidney or perirenal space are sparse..$^{3,4}$ In one other reported case, actinomycosis affected the kidney itself, presenting as a necrotic renal mass that was presumed to be malignancy. The diagnosis of actinomycosis was made only after nephrectomy. ${ }^{3}$ In a third case, the diagnosis was confirmed after Actinomyces was found on an intraoperative frozen section biopsy during a planned partial nephrectomy. ${ }^{4}$

In general, abdominal and retroperitoneal actinomycosis most commonly presents with vague symptoms, including flank or abdominal pain, fever, leukocytosis, and weight loss. The most common imaging modalities obtained for such patients tend to be CT or MRI; these typically reveal a poorly defined, avascular, necrotic mass that is commonly interpreted as either malignancy or abscess. Our patient had a similar presentation with chief complaints of prolonged vague abdominal pain and fevers over a 3-week course, with subsequent workup revealing leukocytosis. Fortunately for our patient, in this case the majority of her abscess burden was located adjacent to the kidney rather than within the parenchyma itself.

Although diagnosis is often delayed in patients with abdominal or retroperitoneal actinomycosis, there is a typically good response to treatment with antibiotic therapy alone once it is made. ${ }^{1}$ The mainstay of treatment is a 2- to 6-week course of parenteral antibiotic therapy with a beta lactam, typically IV penicillin, followed by a prolonged course of oral beta-lactam therapy. There is no consensus on the duration of oral therapy, but most authors agree that a 6-month course of oral penicillin or amoxicillin is a reasonable treatment regimen. ${ }^{1}$ 


\section{Conclusion}

In this article, we present the rare case of a patient with retroperitoneal actinomycosis in the setting of an obstructing ureteral stone. The presumed etiology of her actinomycosis was seeding of normal genitourinary flora via tissue necrosis or forniceal extravasation from the obstructing stone in the setting of chronic malnutrition and prior IUD placement. Proper diagnosis of actinomycosis is the most important factor in management, as associated abscesses are readily treatable with drainage and prolonged beta-lactam therapy.

\section{Disclosure Statement}

No competing financial interests exist.

\section{References}

1. Valour F, Sénéchal A, Dupieux C, et al. Actinomycosis: Etiology, clinical features, diagnosis, treatment, and management. Infect Drug Resist 2014;7:183-197.

2. Bonnefond S, Catroux M, Melenotte C, et al. Clinical features of actinomycosis: A retrospective, multicenter study of 28 cases of miscellaneous presentations. Medicine (Baltimore) 2016;95:e3923.

3. Dieckmann KP, Henke RP, Ovenbeck R. Renal actinomycosis mimicking renal carcinoma. Eur Urol 2001;39: 357-359.
4. Khalaff H, Srigley JR, Klotz LH. Recognition of renal actinomycosis: Nephrectomy can be avoided. Report of a case. Can J Surg 1995;38:77-79.

Address correspondence to:

Michael S. Borofsky, MD

Department of Urology

University of Minnesota Medical School

420 Delaware Street SE, Box 394 Mayo

Minneapolis, MN 55455

E-mail: mborofsk@umn.edu

Cite this article as: Bearrick E, Dixon CA, Rhein J, Borofsky MS (2017) Retroperitoneal actinomycosis: a rare sequela of an infected obstructing ureteral stone, Journal of Endourology Case Reports 3:1, 93-96, DOI: 10.1089/ cren.2017.0048.

\section{Abbreviations Used \\ IUD $=$ intrauterine devices \\ $\mathrm{IV}=$ intravenous}

